\title{
reviscafuences
}

ISSN: 1575-7072 | e-ISSN: 2172-7775

Páginas: 108-124

Recibido: 2020-05-15

Revisado: 2020-09-30

Aceptado: 2020-11-20

Preprint: 2021-01-15

Publicación Final: 2021-01-31 www.revistascientificas.us.es/index.php/fuentes/index

DOI: https://doi.org/10.12795/revistafuentes.2021.v23.i1.12050

\section{Competencia digital docente y el uso de la realidad aumentada en la enseñanza de ciencias en Educación Secundaria Obligatoria}

\section{Digital literacy and the use of augmented reality in teaching science in Secondary Education}

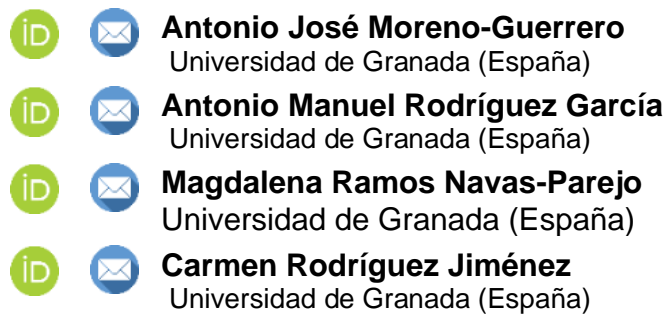

\section{Resumen}

Hoy día la tecnología ocupa un lugar destacado en la sociedad, por lo que es fundamental para desenvolverse en ella con éxito tener adquirida la competencia digital. Los docentes deben desarrollarla de forma adecuada para que su labor educativa sea eficaz y para poder transmitirla a su alumnado. Un ejemplo del uso de la tecnología en educación que se encuentra en auge es la utilización didáctica de la Realidad Aumentada, que ofrece al área de las ciencias una gran cantidad de posibilidades para facilitar el proceso de enseñanza-aprendizaje. El objetivo que se plantea en el presente estudio es conocer la incidencia de la competencia digital en el empleo de la realidad aumentada como recurso didáctico en la impartición de clases relacionadas con las ciencias. El método utilizado es de tipo cuantitativo no experimental, de corte descriptivo, correlacional y con carácter predictivo. La recogida de datos se ha llevado a cabo mediante un cuestionario ad hoc sobre competencia digital docente y realidad aumentada, con diferentes niveles de respuesta. Los resultados muestran que la realidad aumentada es un recurso que ha sido usado por parte de los docentes que imparten ciencias, presentando estos un nivel de competencia digital medio. Se concluye afirmando que los docentes que utilizan la realidad aumentada presentan más competencia digital que aquellos que no lo usan.

\section{Abstract}

Today, technology is a major issue in society, so it is essential to have acquired digital competence in order to be successful in it. An example of the growing use of technology in education is the didactic use of Augmented Reality, which offers the area of science a wealth of possibilities to facilitate the teaching-learning process. The aim of this study is to understand the impact of digital competence on the use of augmented reality as a teaching resource in science-related classes. The method used is quantitative, non-experimental, descriptive, correlational and predictive. The data collection has been carried out through an ad hoc questionnaire on digital teaching competence and augmented reality with different levels of response. The results show that augmented reality is a resource that has been used by teachers teaching science, presenting them with an average level of digital competence. We conclude by stating that teachers who use augmented reality are more digitally competent than those who do not.

\section{Palabras clave / Keywords}

Enseñanza secundaria, competencia profesional, formación de docentes, tecnología educativa, recursos educativos. Secondary education, teacher qualifications, teacher training, educational technology, educational resources. 


\section{Introducción}

Hoy en día los conocimientos y las aptitudes hacia las tecnologías digitales son fundamentales para desenvolverse con éxito en la sociedad (Blayone, 2019). Utilizar las TIC de forma eficiente, independiente y responsable es un requisito clave para la integración social y económica, por lo que se conciben como un factor clave en los procesos de enseñanza-aprendizaje (Starkey, 2019). Por ello, cualquier sistema educativo que se precie debe saber adaptar la realidad tecnológica actual a los espacios de aprendizaje y a su normativa educativa (Asongu y Odhiambo, 2019; Avidov-Ungar y Hanin-Itzak, 2019), lo que precisa, a su vez, de un fuerte despliegue logístico, formativo y económico (Fuentes-Cabrera et al., 2019).

EI uso de las TIC en la sociedad actual obliga al desarrollo de una serie de habilidades, entre las que se encuentra la competencia digital, la cual se define como la capacidad de usar de forma segura, crítica y creativa los recursos tecnológicos, con el objetivo de responder de la forma más eficaz a las exigencias laborales, de aprendizaje y de ocio (Canfarotta y Casado-Muñoz, 2019). Dicha necesidad social se ve reflejada en el ámbito educativo, siendo necesario formar a los alumnos en el desarrollo de esta competencia (Juhanak et al., 2019), por lo que se precisa de la competencia digital docente, la cual hace referencia al conjunto de saberes y destrezas necesarios para el buen uso de las TIC en el ámbito educativo (PrendesEspinosa et al., 2018).

La enseñanza de la competencia digital es un proceso que debe ir en progresión y ser recurrente, para que se genere un conocimiento completo y actualizado (Hsu et al., 2019) en torno a cinco áreas (Olofsson et al., 2019; Rodríguez-García, 2019): 1) Alfabetización en información y datos; 2) Comunicación y colaboración; 3) Creación de contenido digital; 4) Seguridad; y 5) Resolución de problemas. El hecho de poseer diferentes dimensiones la hace compleja, surgiendo incluso la necesidad de organizar y sistematizar la formación de los docentes y su evaluación de forma estandarizada (Cuartero et al., 2019). Además, se compone de dos vertientes: la competencia digital necesaria para facilitar la labor de enseñanza-aprendizaje con el uso de las TIC, y la que tiene que ver con la transmisión al alumnado de los conocimientos fundamentales para que alcancen ellos mismos esta competencia digital (Fernández-Batanero et al., 2019).

En este entramado de la competencia digital, el hecho de utilizar la tecnología de forma creativa se constata como una de las habilidades esenciales que conforman la competencia digital en su globalidad. En esta línea, encontramos en la realidad aumentada (en adelante RA) un potente recurso educativo que cada vez está generando mayor incidencia en los procesos de enseñanza-aprendizaje (Fuentes-Cabrera et al., 2019). La tecnología móvil, por tanto, se ha concebido en la actualidad como una herramienta muy eficaz que apoya el proceso didáctico (Ewais y Troyer, 2019; Tomara y Gouscos, 2019).

Esta tecnología permite combinar información digital y física en tiempo real, generando así una nueva realidad más completa (Bhagat et al., 2019). Estas dos realidades se superponen en distintas capas de información de formatos diversos: imágenes, vídeos, animaciones, etc. El resultado son unos recursos enriquecidos que suponen para el estudiante un aumento de la motivación y del rendimiento académico (Tzima et al., 2019), además de eliminar información innecesaria (Villalustre et al., 2019), aumentar la información de la realidad significativa (Hendajani et al., 2019), contextualizar los aprendizajes (García-Bonete et al., 2019), y generar entornos activos de enseñanza (Barroso-Osuna et al., 2019).

Utilizar la RA en la enseñanza de las ciencias junto con metodologías didácticas activas, acercan el aprendizaje del alumnado a la experimentación (Ewais y Troyer, 2019), facilita la simulación (Safadel y White, 2019) y comprensión de conceptos científicos (Mustami et al., 2019; Tomara y Gouscos, 2019), creando entornos de aprendizaje estimulantes que incrementan la motivación (Kularbphettong y Puengpo, 2018) y la curiosidad por descubrir la ciencia (Chen, 2019; Erbas y Demirer, 2019), reduciendo así la ansiedad que la enseñanza de esta rama pueda generar (Bos et al., 2019; Chen, 2019; Fuchsova y Korenova, 2019).

A pesar de todo, la formación en competencia digital de los docentes supone un reto (Silva-Quiroz, 2017), debido al carácter multidisciplinar que posee en estos casos (Lázaro et al., 2019), así como a la falta de una formación adecuada al respecto, especialmente con los recursos tecnológicos más novedosos (Hilli, 2019). Por este motivo, se hacen necesarios programas de formación continua orientados a su mejora (Falcó-Boudet, 2017), puesto que el desarrollo de la misma se concibe como una clave para el correcto desempeño de la profesión y para la mejora de la calidad institucional de los centros educativos (Gisbert-Cervera y LázaroCantabrana, 2015). 


\section{Metodología}

Esta investigación tiene por objetivo conocer la incidencia de la competencia digital en el empleo de la realidad aumentada como recurso didáctico en la impartición de clases relacionadas con las ciencias. De igual modo, este trabajo pretende dar respuesta a las siguientes preguntas de investigación: ¿hacen uso los profesores de ciencias de la realidad aumentada?, ¿cuáles son los motivos -en el caso de que no la usen- de no utilizar la realidad aumentada?, ¿cuál es el nivel de competencia digital de los docentes?, ¿influye la frecuencia de utilización de la realidad aumentada en las diferentes dimensiones de la competencia digital?

Para la consecución de todo ello se ha desarrollado un estudio cuantitativo, no experimental, de corte descriptivo, correlacional y con carácter predictivo (Hernández et al., 2014).

\subsection{Participantes}

La muestra participante en este estudio asciende a un total de $n=2348$ docentes de Educación Secundaria Obligatoria (ESO) de la comunidad autónoma de Andalucía (España) que imparten alguna asignatura relacionada con las Ciencias Experimentales. Para la consecución de esta cifra se ha seguido una técnica de muestreo no probabilística y por conveniencia, puesto que se ha tenido fácil acceso a los sujetos a través de reuniones y demás rutinas docentes (claustros, reuniones de departamento, consejo escolar, etc.). A su vez, se realizó un llamamiento a la participación a través de redes sociales (Linkedln, Facebook, Twitter, WhatsApp y Telegram) y mediante correo electrónico. De igual modo, la participación se incrementó siguiendo la técnica bola de nieve, mediante la cual los sujetos iniciales participantes en el estudio recomiendan la participación en el mismo a otras personas afines a ellos.

De la cifra anteriormente mencionada, el $47,19 \%$ son hombres $(n=1108)$ y el $52,81 \%$ son mujeres $(n=1240)$. Respecto a la edad, el $36,5 \%$ oscila entre 21 y 30 años ( $n=857)$; el $26,8 \%$ entre 31 y 40 ( $n=617)$; el 25,47\% entre 41 y 50 años $(n=598)$ y, finalmente, un $25,74 \%$ estaría compuesto por profesionales de más de 50 años $(n=276)$.

\subsection{Instrumento y variables de investigación}

La recogida de datos se llevó a cabo mediante un cuestionario ad hoc sobre competencia digital docente y realidad aumentada con diferentes niveles de respuesta: algunas cuestiones son de naturaleza cerrada, dicotómicas y de opinión abierta; sin embargo, la gran mayoría de las preguntas se presentan con una opción de respuesta de escala Likert de 6 puntos, siendo los niveles: A1 (Básico-Bajo), A2 (Básico-Alto), B1 (MedioBajo), B2 (Medio-Alto), C1 (Avanzado-Bajo) y C2 (Avanzado-Alto). El diseño del mismo ha surgido de otros instrumentos ya validados que han sido recogidos de la literatura científica (Agreda et al., 2016; INTEF, 2017; Instefjor y Munthe, 2017; Falcó-Bourdet, 2017; Rodríguez-García, 2019). El cuestionario consta de un total de 84 ítems dividido en seis dimensiones.

\section{Tabla 1}

Dimensiones y variables del cuestionario ad hoc

\begin{tabular}{|l|l|}
\hline Siglas & \\
\hline DSD & Dimensión sociodemográfica \\
\hline REAU & Frecuencia de utilización de la realidad aumentada \\
\hline FCRA & Falta de confianza hacia el enfoque de realidad aumentada \\
\hline ENCA & Enfoque no compatible con el alumnado \\
\hline DFCD & Déficit formativo en competencia digital \\
\hline ART & Ausencia de recursos tecnológicos \\
\hline NTIC & Negativa hacia el uso de las TIC en el proceso formativo \\
\hline CD1 & Dimensión Información y Alfabetización informacional \\
\hline NBFI & Navegación, búsqueda, filtrado de información, datos y contenidos digitales \\
\hline
\end{tabular}




\begin{tabular}{|l|l|}
\hline IDCD & Información, datos y contenidos digitales \\
\hline ARID & Almacenamiento, recuperación de datos y contenidos digitales \\
\hline CD2 & Dimensión Comunicación y Colaboración \\
\hline IMTD & Interacción mediante las tecnologías digitales \\
\hline CICD & Compartir información y contenidos digitales \\
\hline PCL & Participación en línea \\
\hline CMCD & Colaboración mediante canales digitales \\
\hline NETI & Netiqueta \\
\hline GIDI & Gestión de la identidad digital \\
\hline CD3 & Dimensión Creación de Contenidos Digitales \\
\hline DCDI & Desarrollo de contenidos digitales \\
\hline IRCD & Integración y reelaboración de contenidos digitales \\
\hline DALI & Derechos de autor y licencias \\
\hline PROG & Programación \\
\hline CD4 & Dimensión de Seguridad \\
\hline PRDI & Protección de dispositivos \\
\hline PDPE & Protección de datos personales e identidad digital \\
\hline PRSA & Protección de salud \\
\hline PREN & Protección del entorno \\
\hline CD5 & Resolución de Problemas \\
\hline REPT & Resolución de problemas técnicos \\
\hline INRT & Identificación de necesidades y respuesta tecnológica \\
\hline IUTD & Innovación y uso de la tecnología digital de forma creativa \\
\hline ILCD & Identificación de lagunas en la competencia digital \\
\hline
\end{tabular}

La validación del cuestionario se produjo mediante validez de contenido y validez de constructo, siguiendo los preceptos de López-Gómez (2018), Salcines y González (2016) y Santos et al. (2017). La primera se efectuó a través del método Delphi, conformado por diez expertos, los cuales - tras analizar el instrumentootorgaron una valoración media elevada de la herramienta $(\mathrm{M}=4.97, \mathrm{DT}=.41$, mín=1, máx=6), con un puntaje en la de validez de contenidos pertinente (IVCG =.91). Del mismo modo, los especialistas brindaron una serie de recomendaciones con el objetivo de mejorar el cuestionario. Estas estaban enfocadas a la estructura y el uso de determinados conceptos clave. Posteriormente, se realizaron los estadísticos Kappa de Fleiss y W de Kendall, para comprobar la conveniencia y concordancia de los juicios emitidos por los expertos, resultando ambas pruebas adecuadas $(\mathrm{K}=.79 ; \mathrm{W}=.87)$.

Para la validez de constructo, se efectuó un análisis factorial exploratorio, por medio de la técnica de componentes principales con una rotación oblicua, siguiendo el método de Oblimin directo. La adecuación muestral se obtuvo con el test de Kaiser-Meyer-Olkin, hallando una puntuación adecuada $(\mathrm{KMO}=0.79)$. Así pues, la prueba de esfericidad de Bartlett resultó significativa $(7.719 .51 ; \mathrm{df}=617 ; \mathrm{p}=<$ $.001)$.

Por último, se comprobó la fiabilidad del cuestionario a través de medidas de coherencia —en una muestra independiente de 234 participantes - obteniendo valores adecuados de consistencia interna en los estadísticos alfa de Cronbach $(\alpha=0.85)$, fiabilidad compuesta $(F C=0.84)$ y varianza media extractada (VME=0.77), según Tavakol y Dennick (2011). 


\subsection{Análisis}

El análisis de los datos se ha realizado con SPSS 24.0 a través de estadísticos básicos como la media (M) y la desviación típica (DT). Además, se han llevado a cabo pruebas específicas para establecer la propensión de la distribución como el coeficiente de asimetría de Pearson (CAP) y el de apuntamiento de Fisher (CAF). La asociación de las variables se ha efectuado mediante el test Chi-cuadrado de Pearson (X2), junto con las pruebas $\mathrm{V}$ de Cramer (V) y coeficiente de contingencia (Cont), para encontrar la fuerza de relación entre las variables estudiadas. Asimismo, también se ha realizado un modelo de regresión lineal múltiple con el objetivo de pronosticar el efecto que a nivel individual cada dimensión de cada una de las áreas de la competencia digital docente (variables independientes) tienen sobre la frecuencia de uso del flipped learning (variable dependiente). La significancia estadística ha sido tomada a partir de $p<.05$

\section{Resultados}

A lo largo de este epígrafe se presentan los resultados obtenidos de los diversos análisis aplicados a las variables planteadas. Según se muestra en la Figura 1, el $60.35 \%$ de los participantes en el estudio han utilizado, en diversa medida, la realidad aumentada durante el proceso de enseñanza y aprendizaje, frente al $39.65 \%$ de los docentes que no han hecho uso de ella en sus planteamientos didácticos. Estas cifras muestran que la realidad aumentada es un recurso de interés para los docentes.

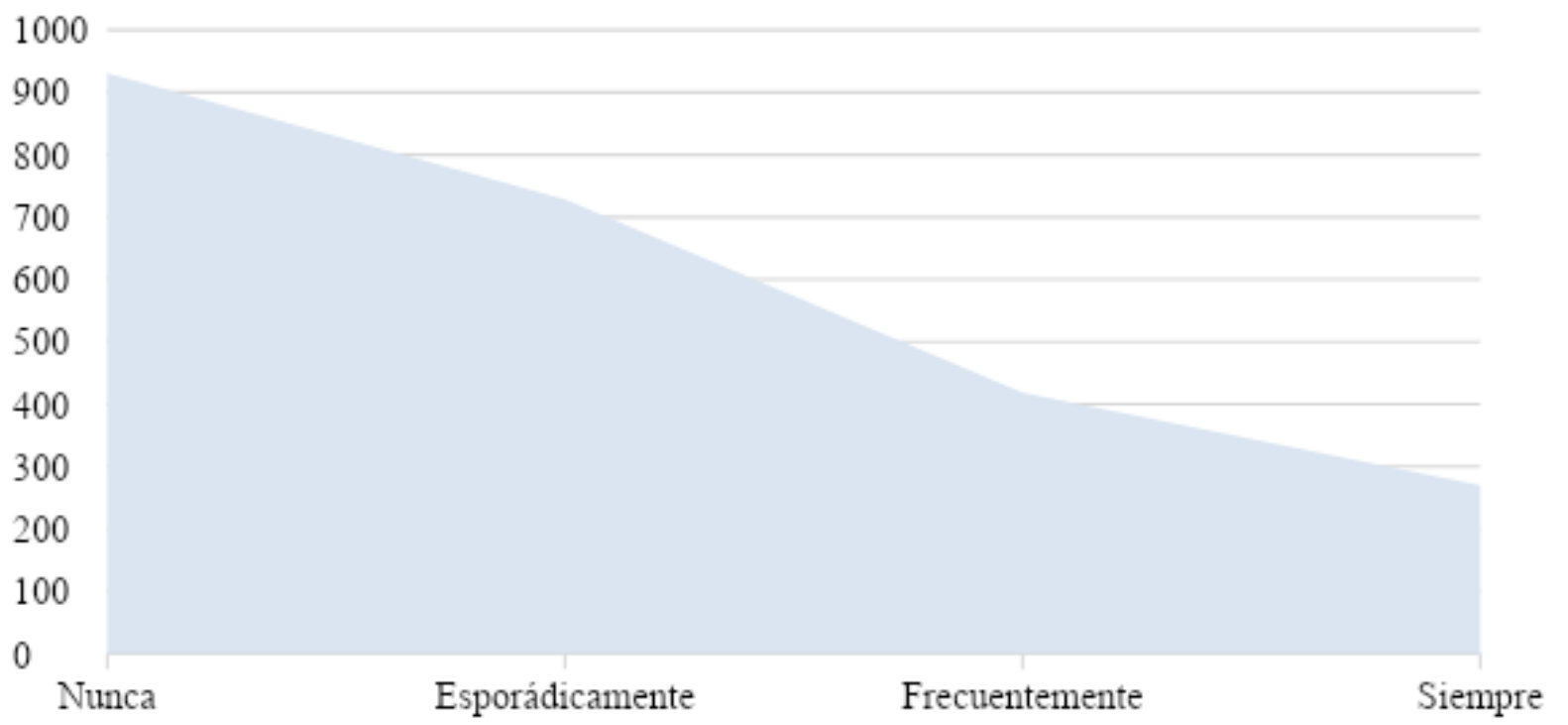

Figura 1. Utilización de la realidad aumentada por parte del profesorado.

Centrando el análisis en aquellos docentes que no han utilizado la realidad aumentada en los procesos de enseñanza, se observa como principal causa la falta de recursos tecnológicos seguido del déficit que presentan con respecto a la competencia digital docente. En menor medida, la negativa ante el uso de las TIC, el no ser un enfoque compatible para el alumnado y la falta de confianza en el uso de la realidad aumentada en los procesos de enseñanza y aprendizaje son también motivos para su falta de uso. 


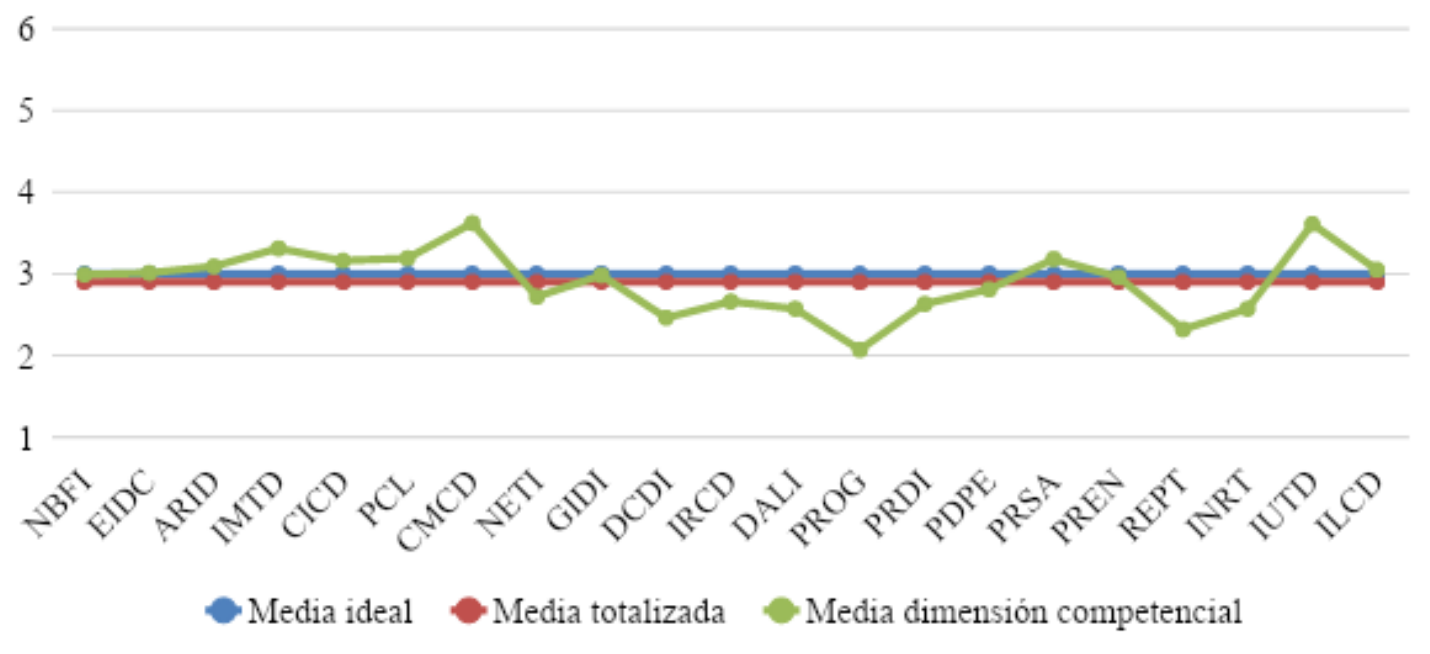

Figura 2. Motivos por los que el profesorado no utiliza la realidad aumentada.

Analizando la competencia digital de todos los docentes que han participado en el estudio, se observa que presentan un nivel competencial medio, habiendo déficits competenciales en la creación de contenidos digitales (CD3), principalmente, dado que todas sus variables de sitúan en un nivel medio-bajo. El resto de áreas competenciales presentan variables que se sitúan en niveles medio-bajo o medio-alto. No se observan ni niveles muy superiores ni muy inferiores en ninguno de los ítems analizados, situándose todos ellos en una horquilla media entre 2 y 4 . El área competencial con mayor nivel se refiere a la colaboración mediante canales digitales y, al contrario, el más bajo es el referente a la programación (Tabla 2).

\section{Tabla 2}

Descriptivo de las áreas competenciales

\begin{tabular}{|c|c|c|c|c|c|c|c|c|c|c|}
\hline & \multicolumn{6}{|c|}{ Escala Likert $\boldsymbol{n} / \%$} & \multicolumn{4}{|c|}{ Parámetros } \\
\hline & A1 & A2 & B1 & B2 & C1 & C2 & $M$ & DT & CAP & CAF \\
\hline \multicolumn{11}{|l|}{ CD1 } \\
\hline \multirow[t]{2}{*}{ NBFI } & 285 & 628 & 731 & 357 & 213 & 134 & 2.99 & 1.334 & 1.494 & -.0333 \\
\hline & 12.14 & 26.75 & 31.13 & 15.2 & 9.07 & 5.71 & & & & \\
\hline \multirow[t]{2}{*}{ IDCD } & 317 & 579 & 694 & 416 & 194 & 148 & 3.01 & 1.364 & 1.476 & -0.419 \\
\hline & 13.5 & 24.66 & 29.56 & 17.72 & 8.26 & 6.3 & & & & \\
\hline \multirow[t]{2}{*}{ ARID } & 150 & 671 & 792 & 376 & 266 & 93 & 3.09 & 1.225 & 1.706 & -0.310 \\
\hline & 6.39 & 28.58 & 33.73 & 16.01 & 11.33 & 3.96 & & & & \\
\hline \multicolumn{11}{|l|}{ CD2 } \\
\hline \multirow[t]{2}{*}{ IMTD } & 114 & 511 & 836 & 489 & 194 & 204 & 3.31 & 1.276 & 1.816 & -0.269 \\
\hline & 4.86 & 21.76 & 35.6 & 20.83 & 8.26 & 8.69 & & & & \\
\hline \multirow[t]{2}{*}{ CICD } & 138 & 493 & 991 & 407 & 203 & 116 & 3.16 & 1.175 & 1.843 & 0.108 \\
\hline & 5.88 & 21 & 42.21 & 17.33 & 8.65 & 4.94 & & & & \\
\hline \multirow[t]{2}{*}{$\mathrm{PCL}$} & 182 & 337 & 1057 & 483 & 181 & 108 & 3.19 & 1.158 & 1.898 & 0.241 \\
\hline & 7.75 & 14.35 & 45.02 & 20.57 & 7.71 & 4.6 & & & & \\
\hline \multirow[t]{2}{*}{ CMCD } & 74 & 218 & 736 & 849 & 418 & 53 & 3.62 & 1.048 & 2.507 & -0.056 \\
\hline & 3.15 & 9.28 & 31.35 & 36.16 & 17.8 & 2.26 & & & & \\
\hline \multirow[t]{2}{*}{ NETI } & 416 & 729 & 573 & 386 & 197 & 47 & 2.72 & 1.274 & 1.355 & -0.488 \\
\hline & 17.72 & 31.05 & 24.4 & 16.44 & 8.39 & 2 & & & & \\
\hline \multirow[t]{2}{*}{ GIDI } & 322 & 698 & 497 & 493 & 208 & 130 & 2.98 & 1.381 & 1.434 & -0.615 \\
\hline & 13.71 & 29.73 & 21.71 & 21 & 8.86 & 5.54 & & & & \\
\hline \multicolumn{11}{|l|}{ CD3 } \\
\hline \multirow[t]{2}{*}{ DCDI } & 571 & 778 & 539 & 294 & 117 & 49 & 2.46 & 1.239 & 1.186 & 0.028 \\
\hline & 24.32 & 33.13 & 22.96 & 12.52 & 4.98 & 2.09 & & & & \\
\hline \multirow[t]{2}{*}{ IRCD } & 524 & 661 & 592 & 317 & 149 & 105 & 2.66 & 1.362 & 1.224 & -0.211 \\
\hline & 22.32 & 28.15 & 25.21 & 13.5 & 6.35 & 4.47 & & & & \\
\hline \multirow[t]{2}{*}{ DALI } & 861 & 408 & 396 & 354 & 216 & 113 & 2.57 & 1.552 & 1.012 & -0.806 \\
\hline & 36.67 & 17.38 & 16.87 & 15.08 & 9.2 & 4.81 & & & & \\
\hline
\end{tabular}




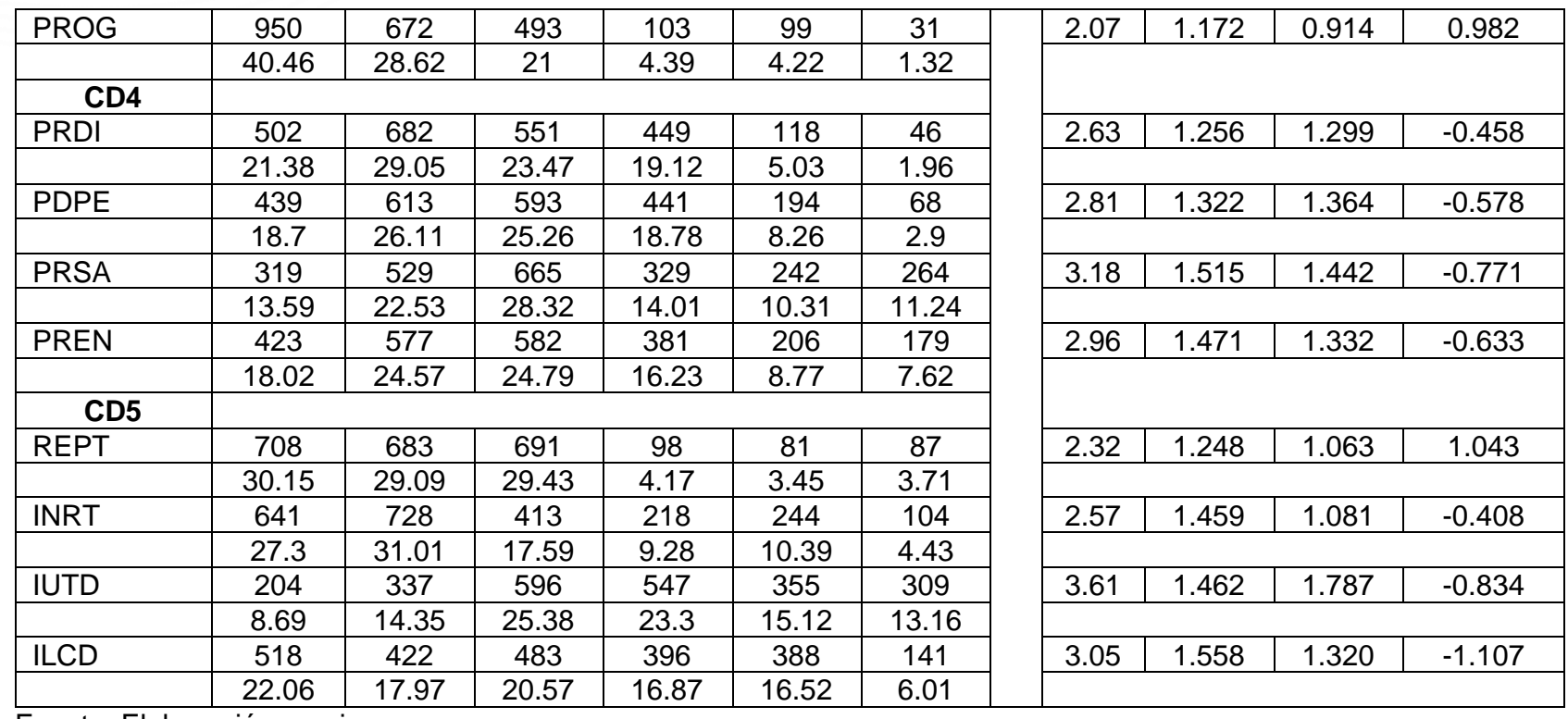

Fuente: Elaboración propia.

Si se analiza la Figura 3, se observa -en los docentes que imparten ciencias-como la media totalizada se encuentra en un nivel inferior a la media ideal, aunque la diferencia entre ambas es mínima. Tal y como se muestra, la dimensión en comunicación y colaboración presenta varios ítems por encima de la media, mientras que la dimensión de creación de contenidos digitales se encuentra por debajo de la media.

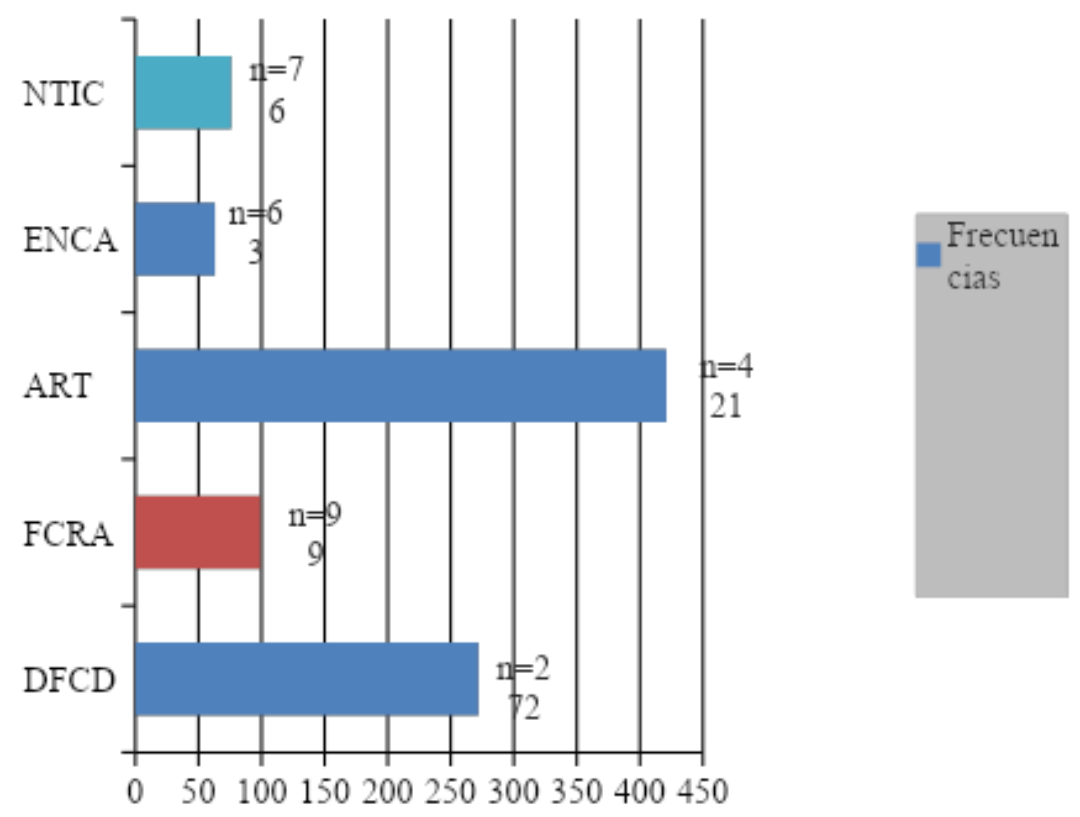

Figura 3. Motivos por los que el profesorado no usa la realidad aumentada.

Analizando la relación existente entre el uso de la realidad aumentada en los procesos de enseñanza y aprendizaje (Tabla 3) con respecto a la dimensión de información y alfabetización informacional, se muestran correlaciones significativas entre todos sus ítems. Es decir, en las variables navegación, búsqueda, filtrado de información, datos y contenidos digitales; información, datos y contenidos digitales; y almacenamiento, recuperación de datos y contenidos digitales. La fuerza de relación existente entre ellos es baja según el estadístico V de Cramer (<.02). 
Tabla 3

Correlación entre REAU y CD1

\begin{tabular}{|c|c|c|c|c|c|c|c|c|c|c|}
\hline \multirow[t]{2}{*}{ DFCD } & \multicolumn{6}{|c|}{ Escala Likert $n / \%$} & \multicolumn{4}{|c|}{ Parámetros } \\
\hline & A1 & A2 & B1 & B2 & C1 & C2 & $x^{2}(g l)$ & $p$-va. & Con & $\mathrm{V}$ \\
\hline \multicolumn{7}{|l|}{ NBFI } & $162.89(15)$ & $<.000$ & .255 & .152 \\
\hline \multirow[t]{2}{*}{ NUN } & 86 & 202 & 334 & 114 & 102 & 93 & & & & \\
\hline & 3.66 & 8.6 & 14.22 & 4.86 & 4.34 & 3.96 & & & & \\
\hline \multirow[t]{2}{*}{ ESP } & 73 & 231 & 220 & 133 & 56 & 15 & & & & \\
\hline & 3.11 & 9.84 & 9.37 & 5.66 & 2.39 & 0.64 & & & & \\
\hline \multirow[t]{2}{*}{ FRE } & 57 & 123 & 103 & 86 & 33 & 17 & & & & \\
\hline & 2.43 & 5.24 & 4.39 & 3.66 & 1.41 & 0.72 & & & & \\
\hline \multirow[t]{2}{*}{ SIE } & 69 & 72 & 74 & 24 & 22 & 9 & & & & \\
\hline & 2.94 & 3.07 & 3.15 & 1.02 & 0.94 & 0.38 & & & & \\
\hline \multicolumn{7}{|l|}{ IDCD } & 119.39(15) & $<.000$ & .220 & .130 \\
\hline \multirow[t]{2}{*}{$\mathrm{NUN}$} & 94 & 197 & 318 & 147 & 89 & 86 & & & & \\
\hline & 4 & 8.39 & 13.54 & 6.26 & 3.79 & 3.66 & & & & \\
\hline \multirow[t]{2}{*}{ ESP } & 86 & 203 & 215 & 143 & 54 & 27 & & & & \\
\hline & 3.66 & 8.65 & 9.16 & 609 & 2.3 & 1.15 & & & & \\
\hline \multirow[t]{2}{*}{ FRE } & 61 & 117 & 103 & 85 & 24 & 29 & & & & \\
\hline & 2.6 & 4.98 & 4.39 & 3.62 & 1.02 & 1.24 & & & & \\
\hline \multirow[t]{2}{*}{ SIE } & 76 & 62 & 58 & 41 & 27 & 6 & & & & \\
\hline & 3.24 & 2.64 & 2.47 & 1.75 & 1.15 & 0.26 & & & & \\
\hline \multicolumn{7}{|l|}{ ARID } & $58.98(15)$ & $<.000$ & .157 & .092 \\
\hline \multirow[t]{2}{*}{ NUN } & 62 & 256 & 328 & 129 & 109 & 47 & & & & \\
\hline & 2.64 & 10.9 & 13.97 & 5.49 & 4.64 & 2 & & & & \\
\hline \multirow[t]{2}{*}{ ESP } & 43 & 213 & 268 & 113 & 64 & 27 & & & & \\
\hline & 1.83 & 9.07 & 11.41 & 4.81 & 2.73 & 1.15 & & & & \\
\hline \multirow[t]{2}{*}{ FRE } & 29 & 137 & 119 & 83 & 36 & 15 & & & & \\
\hline & 1.24 & 5.83 & 5.07 & 3.53 & 1.53 & 0.64 & & & & \\
\hline \multirow[t]{2}{*}{ SIE } & 16 & 65 & 77 & 51 & 57 & 4 & & & & \\
\hline & 0.68 & 2.77 & 3.28 & 2.17 & 2.43 & 0.17 & & & & \\
\hline
\end{tabular}

Nota: $\mathrm{NUN}=$ Nunca; $\mathrm{ESP}=$ Esporádico; $\mathrm{FRE}=$ Frecuente; $\mathrm{SIE}=$ Siempre.

En la dimensión de comunicación y colaboración, las relaciones entre el uso de la realidad aumentada y los ítems de la competencia digital docente (Tabla 4), son significativas. Específicamente en las variables interacción mediante las tecnologías digitales; compartir información y contenidos digitales; participación en línea; colaboración mediante canales digitales; netiqueta; y gestión de la identidad digital. La fuerza de relación es baja (<.02), exceptuando la variable compartir información y contenidos digitales, donde la relación es media $(.02<\mathrm{ES}<.06)$.

\section{Tabla 4}

Correlación entre REAU y CD2

\begin{tabular}{|c|c|c|c|c|c|c|c|c|c|c|}
\hline \multirow[t]{2}{*}{ DFCD } & \multicolumn{6}{|c|}{ Escala Likert $n / \%$} & \multicolumn{4}{|c|}{ Parámetros } \\
\hline & A1 & A2 & B1 & B2 & C1 & C2 & $x^{2}(g l)$ & $p$-va. & Con & $\mathbf{v}$ \\
\hline IMTD & & & & & & & $54,01(15)$ & $<.000$ & .150 & .088 \\
\hline
\end{tabular}




\begin{tabular}{|c|c|c|c|c|c|c|c|c|c|c|}
\hline \multirow[t]{2}{*}{ NUN } & 51 & 182 & 368 & 170 & 74 & 86 & & & & \\
\hline & 2.17 & 7.75 & 15.67 & 7.24 & 3.15 & 3.66 & & & & \\
\hline \multirow[t]{2}{*}{ ESP } & 28 & 171 & 272 & 164 & 53 & 40 & & & & \\
\hline & 1.19 & 7.28 & 11.58 & 6.98 & 2.26 & 1.7 & & & & \\
\hline \multirow[t]{2}{*}{ FRE } & 23 & 105 & 109 & 104 & 38 & 40 & & & & \\
\hline & 0.98 & 4.47 & 4.64 & 4.43 & 1.62 & 1.7 & & & & \\
\hline \multirow[t]{2}{*}{ SIE } & 12 & 53 & 87 & 51 & 29 & 38 & & & & \\
\hline & 0.51 & 2.26 & 3.71 & 2.17 & 1.24 & 1.62 & & & & \\
\hline \multicolumn{7}{|l|}{ CICD } & $292.02(15)$ & $<.000$ & .333 & .204 \\
\hline \multirow[t]{2}{*}{ NUN } & 26 & 196 & 281 & 207 & 129 & 92 & & & & \\
\hline & 1.11 & 8.35 & 11.97 & 8.82 & 5.49 & 3.92 & & & & \\
\hline \multirow[t]{2}{*}{ ESP } & 32 & 144 & 395 & 106 & 30 & 21 & & & & \\
\hline & 1.36 & 6.13 & 16.82 & 4.51 & 1.28 & 0.89 & & & & \\
\hline \multirow[t]{2}{*}{ FRE } & 38 & 82 & 198 & 63 & 36 & 2 & & & & \\
\hline & 1.62 & 3.49 & 8.43 & 2.68 & 1.53 & 0.09 & & & & \\
\hline \multirow[t]{2}{*}{ SIE } & 42 & 71 & 117 & 31 & 8 & 1 & & & & \\
\hline & 1.79 & 3.02 & 4.98 & 1.32 & 0.34 & 0.04 & & & & \\
\hline \multicolumn{7}{|l|}{ PCL } & $238.04(15)$ & $<.000$ & .303 & .184 \\
\hline \multirow[t]{2}{*}{ NUN } & 37 & 144 & 316 & 263 & 89 & 82 & & & & \\
\hline & 1.58 & 6.13 & 13.46 & 11.2 & 3.79 & 3.49 & & & & \\
\hline \multirow[t]{2}{*}{ ESP } & 54 & 82 & 380 & 121 & 72 & 19 & & & & \\
\hline & 2.3 & 3.49 & 16.18 & 5.15 & 3.07 & 0.81 & & & & \\
\hline \multirow[t]{2}{*}{ FRE } & 48 & 71 & 211 & 71 & 14 & 4 & & & & \\
\hline & 2.08 & 3.02 & 8.99 & 3.02 & 0.6 & 0.17 & & & & \\
\hline \multirow[t]{2}{*}{ SIE } & 43 & 40 & 150 & 28 & 6 & 3 & & & & \\
\hline & 1.83 & 1.7 & 6.39 & 1.19 & 0.26 & 0.13 & & & & \\
\hline \multicolumn{7}{|l|}{ CMCD } & 205.19(15) & $<.000$ & .283 & .171 \\
\hline \multirow[t]{2}{*}{ NUN } & 15 & 96 & 214 & 383 & 181 & 42 & & & & \\
\hline & 0.64 & 4.09 & 9.11 & 16.31 & 7.71 & 1.79 & & & & \\
\hline \multirow[t]{2}{*}{ ESP } & 24 & 59 & 275 & 221 & 145 & 4 & & & & \\
\hline & 1.02 & 2.51 & 11.71 & 9.41 & 6.18 & 0.17 & & & & \\
\hline \multirow[t]{2}{*}{ FRE } & 24 & 41 & 199 & 97 & 54 & 4 & & & & \\
\hline & 1.02 & 1.75 & 8.48 & 4.13 & 2.3 & 0.17 & & & & \\
\hline \multirow[t]{2}{*}{ SIE } & 11 & 22 & 48 & 148 & 38 & 3 & & & & \\
\hline & 0.47 & 0.94 & 2.04 & 6.3 & 1.62 & 0.13 & & & & \\
\hline \multicolumn{7}{|l|}{ NETI } & $116.23(15)$ & $<.000$ & .217 & .128 \\
\hline \multirow[t]{2}{*}{ NUN } & 115 & 299 & 214 & 171 & 103 & 29 & & & & \\
\hline & 4.9 & 12.73 & 9.11 & 7.28 & 4.39 & 1.24 & & & & \\
\hline \multirow[t]{2}{*}{ ESP } & 124 & 261 & 164 & 120 & 47 & 12 & & & & \\
\hline & 5.28 & 11.12 & 6.98 & 5.11 & 2 & 0.51 & & & & \\
\hline FRE & 124 & 114 & 99 & 53 & 23 & 6 & & & & \\
\hline
\end{tabular}




\begin{tabular}{|c|c|c|c|c|c|c|c|c|c|c|}
\hline & 5.28 & 4.86 & 4.22 & 2.26 & 0.98 & 0.26 & & \multirow[b]{4}{*}{$<.000$} & \multirow[b]{4}{*}{.207} & \multirow[b]{4}{*}{.122} \\
\hline \multirow[t]{2}{*}{ SIE } & 53 & 55 & 96 & 42 & 24 & 0 & \multirow[b]{3}{*}{$105.26(15)$} & & & \\
\hline & 2.26 & 2.34 & 4.09 & 1.79 & 1.02 & 0 & & & & \\
\hline \multicolumn{7}{|l|}{ GIDI } & & & & \\
\hline \multirow[t]{2}{*}{ NUN } & 89 & 285 & 186 & 217 & 110 & 44 & & & & \\
\hline & 3.79 & 12.14 & 7.92 & 9.24 & 4.68 & 1.87 & & & & \\
\hline \multirow[t]{2}{*}{ ESP } & 95 & 254 & 124 & 155 & 49 & 51 & & & & \\
\hline & 4.05 & 10.82 & 5.28 & 6.6 & 2.09 & 2.17 & & & & \\
\hline \multirow[t]{2}{*}{ FRE } & 90 & 107 & 99 & 78 & 25 & 20 & & & & \\
\hline & 3.83 & 4.56 & 4.22 & 3.32 & 1.06 & 0.85 & & & & \\
\hline \multirow[t]{2}{*}{ SIE } & 48 & 52 & 88 & 43 & 24 & 15 & & & & \\
\hline & 2.04 & 2.21 & 3.75 & 1.83 & 1.02 & 0.64 & & & & \\
\hline
\end{tabular}

Nota: NUN=Nunca; ESP=Esporádico; FRE=Frecuente; SIE=Siempre.

En la dimensión de creación de contenidos digitales se alcanzan relaciones significativas a nivel estadístico en todos los ítems que lo componen. Estas variables son desarrollo de contenidos digitales; integración y reelaboración de contenidos digitales; derechos de autor y licencias; programación. La fuerza de relación existente es baja (Tabla 5).

\section{Tabla 5}

Correlación entre REAU y $C D 3$

\begin{tabular}{|c|c|c|c|c|c|c|c|c|c|c|}
\hline \multirow[t]{2}{*}{ DFCD } & \multicolumn{6}{|c|}{ Escala Likert $n / \%$} & \multicolumn{4}{|c|}{ Parámetros } \\
\hline & A1 & A2 & B1 & B2 & C1 & C2 & $x^{2}(g l)$ & $p$-va. & Con & $\mathbf{v}$ \\
\hline \multirow{3}{*}{$\begin{array}{l}\text { DCDI } \\
\text { NUN }\end{array}$} & & & & & & & 100.51(15) & $<.000$ & 203 & .119 \\
\hline & 264 & 301 & 214 & 87 & 43 & 22 & & & & \\
\hline & 11.24 & 12.82 & 9.11 & 3.71 & 1.83 & 0.94 & & & & \\
\hline \multirow[t]{2}{*}{ ESP } & 174 & 207 & 211 & 83 & 39 & 14 & & & & \\
\hline & 7.41 & 8.82 & 8.99 & 3.53 & 1.66 & 0.6 & & & & \\
\hline \multirow[t]{2}{*}{ FRE } & 85 & 146 & 92 & 66 & 21 & 9 & & & & \\
\hline & 3.62 & 6.22 & 3.92 & 2.81 & 0.89 & 0.38 & & & & \\
\hline \multirow[t]{2}{*}{ SIE } & 48 & 124 & 22 & 58 & 14 & 4 & & & & \\
\hline & 2.04 & 5.28 & 0.94 & 2.47 & 0.6 & 0.17 & & & & \\
\hline \multicolumn{7}{|l|}{ IRCD } & $79.01(15)$ & $<.000$ & .180 & .106 \\
\hline \multirow{2}{*}{$\overline{\text { NUN }}$} & 251 & 234 & 213 & 129 & 69 & 35 & & & & \\
\hline & 10.69 & 9.97 & 9.07 & 5.49 & 2.94 & 1.49 & & & & \\
\hline \multirow[t]{2}{*}{ ESP } & 155 & 209 & 215 & 87 & 37 & 25 & & & & \\
\hline & 6.6 & 8.9 & 9.16 & 3.71 & 1.58 & 1.06 & & & & \\
\hline \multirow[t]{2}{*}{ FRE } & 74 & 117 & 121 & 57 & 32 & 18 & & & & \\
\hline & 3.15 & 4.98 & 5.15 & 2.43 & 1.36 & 0.77 & & & & \\
\hline \multirow[t]{2}{*}{ SIE } & 44 & 101 & 43 & 44 & 11 & 27 & & & & \\
\hline & 1.87 & 4.3 & 1.83 & 1.87 & 0.47 & 1.15 & & & & \\
\hline \multicolumn{7}{|l|}{ DALI } & $62.89(15)$ & $<.000$ & .162 & .094 \\
\hline \multirow[t]{2}{*}{ NUN } & 372 & 131 & 132 & 144 & 105 & 47 & & & & \\
\hline & 15.84 & 5.58 & 5.62 & 6.13 & 4.47 & 2 & & & & \\
\hline
\end{tabular}




\begin{tabular}{|c|c|c|c|c|c|c|c|c|c|c|}
\hline \multirow[t]{2}{*}{ ESP } & 266 & 124 & 145 & 104 & 60 & 29 & & & & \\
\hline & 11.33 & 5.28 & 6.18 & 4.43 & 2.56 & 1.24 & & & & \\
\hline \multirow[t]{2}{*}{ FRE } & 112 & 95 & 83 & 70 & 41 & 18 & & & & \\
\hline & 4.77 & 4.05 & 3.53 & 2.98 & 1.75 & 0.77 & & & & \\
\hline \multirow[t]{2}{*}{ SIE } & 111 & 58 & 36 & 36 & 10 & 19 & & & & \\
\hline & 4.73 & 2.47 & 1.53 & 1.53 & 0.43 & 0.81 & & & & \\
\hline \multicolumn{7}{|l|}{ PROG } & $43.43(15)$ & $<.000$ & .135 & .079 \\
\hline \multirow[t]{2}{*}{ NUN } & 405 & 279 & 153 & 39 & 43 & 12 & & & & \\
\hline & 17.25 & 11.88 & 6.52 & 1.66 & 1.83 & 0.51 & & & & \\
\hline \multirow[t]{2}{*}{ ESP } & 307 & 186 & 167 & 32 & 28 & 8 & & & & \\
\hline & 13.07 & 7.92 & 7.11 & 1.36 & 1.19 & 0.34 & & & & \\
\hline \multirow[t]{2}{*}{ FRE } & 124 & 138 & 114 & 19 & 19 & 5 & & & & \\
\hline & 5.28 & 5.88 & 4.86 & 0.81 & 0.81 & 0.21 & & & & \\
\hline \multirow[t]{2}{*}{ SIE } & 114 & 69 & 59 & 13 & 9 & 6 & & & & \\
\hline & 4.86 & 2.94 & 2.51 & 0.55 & 0.38 & 0.26 & & & & \\
\hline
\end{tabular}

Nota: NUN=Nunca; ESP=Esporádico; FRE=Frecuente; SIE=Siempre.

En la dimensión de seguridad, la relación entre el uso de la realidad aumentada y los ítems que componen esta dimensión son significativas. Específicamente en las variables protección de dispositivos; protección de datos personales e identidad digital; protección de salud; y protección del entorno. La fuerza de asociación en todos los casos es baja (Tabla 6).

\section{Tabla 6}

Correlación entre REAU y CD4

\begin{tabular}{|c|c|c|c|c|c|c|c|c|c|c|c|}
\hline \multirow[t]{2}{*}{ c } & \multicolumn{6}{|c|}{ Escala Likert $n / \%$} & \multicolumn{5}{|c|}{ Parámetros } \\
\hline & A1 & A2 & B1 & B2 & C1 & C2 & $x^{2}(g l)$ & $p$-va. & Con & $\mathbf{v}$ & \\
\hline PRDI & & & & & & & $220.37(15)$ & $<.000$ & .293 & .177 & \\
\hline \multirow[t]{2}{*}{ NUN } & 97 & 279 & 214 & 251 & 63 & 27 & & & & & \\
\hline & 4.13 & 11.88 & 9.11 & 10.69 & 2.68 & 1.15 & & & & & \\
\hline \multirow[t]{2}{*}{ ESP } & 189 & 191 & 159 & 155 & 24 & 10 & & & & & \\
\hline & 8.05 & 8.13 & 6.77 & 6.6 & 1.02 & 0.43 & & & & & \\
\hline \multirow[t]{2}{*}{ FRE } & 117 & 138 & 121 & 21 & 19 & 3 & & & & & \\
\hline & 4.98 & 5.88 & 5.15 & 0.89 & 0.81 & 0.13 & & & & & \\
\hline \multirow[t]{2}{*}{ SIE } & 99 & 74 & 57 & 22 & 12 & 6 & & & & & \\
\hline & 4.22 & 3.15 & 2.43 & 0.94 & 0.51 & 0.26 & & & & & \\
\hline \multicolumn{7}{|l|}{ PDPE } & $99.42(15)$ & $<.000$ & .202 & .119 & \\
\hline \multirow[t]{2}{*}{$\overline{N U N}$} & 184 & 254 & 179 & 184 & 106 & 24 & & & & & \\
\hline & 7.84 & 10.82 & 7.62 & 7.84 & 4.51 & 1.02 & & & & & \\
\hline \multirow[t]{2}{*}{ ESP } & 104 & 184 & 267 & 123 & 31 & 19 & & & & & \\
\hline & 4.43 & 7.84 & 11.37 & 5.24 & 1.32 & 0.81 & & & & & \\
\hline \multirow[t]{2}{*}{ FRE } & 90 & 99 & 93 & 86 & 35 & 16 & & & & & \\
\hline & 3.83 & 4.22 & 3.96 & 3.66 & 1.49 & 0.68 & & & & & \\
\hline \multirow[t]{2}{*}{ SIE } & 61 & 76 & 54 & 48 & 22 & 9 & & & & & \\
\hline & 2.6 & 3.24 & 2.3 & 2.04 & 0.94 & 0.38 & & & & & \\
\hline
\end{tabular}




\begin{tabular}{|c|c|c|c|c|c|c|c|c|c|c|}
\hline PRSA & & & & & & & $161.83(15)$ & $<.000$ & .254 & .152 \\
\hline \multirow[t]{2}{*}{ NUN } & 126 & 196 & 206 & 99 & 144 & 160 & & & & \\
\hline & 5.37 & 8.35 & 8.77 & 4.22 & 6.13 & 6.81 & & & & \\
\hline \multirow[t]{2}{*}{ ESP } & 80 & 161 & 279 & 104 & 43 & 61 & & & & \\
\hline & 3.41 & 6.86 & 11.88 & 4.43 & 1.83 & 2.6 & & & & \\
\hline \multirow[t]{2}{*}{ FRE } & 65 & 98 & 115 & 81 & 31 & 29 & & & & \\
\hline & 2.77 & 4.17 & 4.9 & 3.45 & 1.32 & 1.24 & & & & \\
\hline \multirow[t]{2}{*}{ SIE } & 48 & 74 & 65 & 45 & 24 & 14 & & & & \\
\hline & 2.04 & 3.15 & 2.77 & 1.92 & 1.02 & 0.6 & & & & \\
\hline \multicolumn{7}{|l|}{ PREN } & 71.61(15) & $<.000$ & .172 & .101 \\
\hline \multirow[t]{2}{*}{ NUN } & 188 & 203 & 204 & 122 & 119 & 95 & & & & \\
\hline & 8.01 & 8.65 & 8.69 & 5.2 & 5.07 & 4.05 & & & & \\
\hline \multirow[t]{2}{*}{ ESP } & 115 & 196 & 205 & 129 & 39 & 44 & & & & \\
\hline & 4.9 & 8.35 & 8.73 & 5.49 & 1.66 & 1.87 & & & & \\
\hline \multirow[t]{2}{*}{ FRE } & 74 & 101 & 111 & 83 & 27 & 23 & & & & \\
\hline & 3.15 & 4.3 & 4.73 & 3.53 & 1.15 & 0.98 & & & & \\
\hline \multirow[t]{2}{*}{ SIE } & 46 & 77 & 62 & 47 & 21 & 17 & & & & \\
\hline & 1.96 & 3.28 & 2.64 & 2 & 0.89 & 0.72 & & & & \\
\hline
\end{tabular}

Nota: NUN=Nunca; ESP=Esporádico; FRE=Frecuente; $\mathrm{SIE}=$ Siempre.

Finalmente, en la dimensión de resolución de problemas, todas las variables muestran relación de significancia con respecto a la utilización de la realidad aumentada en los procesos formativos. Concretamente en las variables resolución de problemas técnicos; identificación de necesidades y respuesta tecnológica; innovación y uso de la tecnología digital de forma creativa; e identificación de lagunas en la competencia digital. La fuerza de relación es baja (tabla 7).

Tabla 7

Correlación entre REAU y CD5

\begin{tabular}{|c|c|c|c|c|c|c|c|c|c|c|}
\hline \multirow[t]{2}{*}{ DFCD } & \multicolumn{6}{|c|}{ Escala Likert $n / \%$} & \multicolumn{4}{|c|}{ Parámetros } \\
\hline & A1 & A2 & B1 & B2 & C1 & C2 & $x^{2}(g l)$ & $p$-va. & Con & $\mathbf{V}$ \\
\hline & & & & & & & $85.52(15)$ & $<.000$ & .187 & .110 \\
\hline \multirow[t]{2}{*}{ NUN } & 286 & 234 & 290 & 31 & 38 & 52 & & & & \\
\hline & 12.18 & 9.97 & 12.35 & 1.32 & 1.62 & 2.21 & & & & \\
\hline \multirow[t]{2}{*}{ ESP } & 264 & 188 & 219 & 25 & 17 & 15 & & & & \\
\hline & 11.24 & 8.01 & 9.33 & 1.06 & 0.72 & 0.64 & & & & \\
\hline \multirow[t]{2}{*}{ FRE } & 96 & 162 & 116 & 19 & 15 & 11 & & & & \\
\hline & 4.09 & 6.9 & 4.94 & 0.81 & 0.64 & 0.47 & & & & \\
\hline \multirow[t]{2}{*}{ SIE } & 62 & 99 & 66 & 23 & 11 & 9 & & & & \\
\hline & 2.64 & 4.22 & 2.81 & 0.98 & 0.47 & 0.38 & & & & \\
\hline & & & & & & & $103.55(15)$ & $<.000$ & .206 & .121 \\
\hline \multirow{2}{*}{ NUN } & 301 & 331 & 134 & 72 & 61 & 32 & & & & \\
\hline & 12.82 & 14.1 & 5.71 & 3.07 & 2.6 & 1.36 & & & & \\
\hline \multirow[t]{2}{*}{ ESP } & 175 & 217 & 149 & 59 & 93 & 35 & & & & \\
\hline & 7.45 & 9.24 & 6.35 & 2.51 & 3.96 & 1.49 & & & & \\
\hline
\end{tabular}




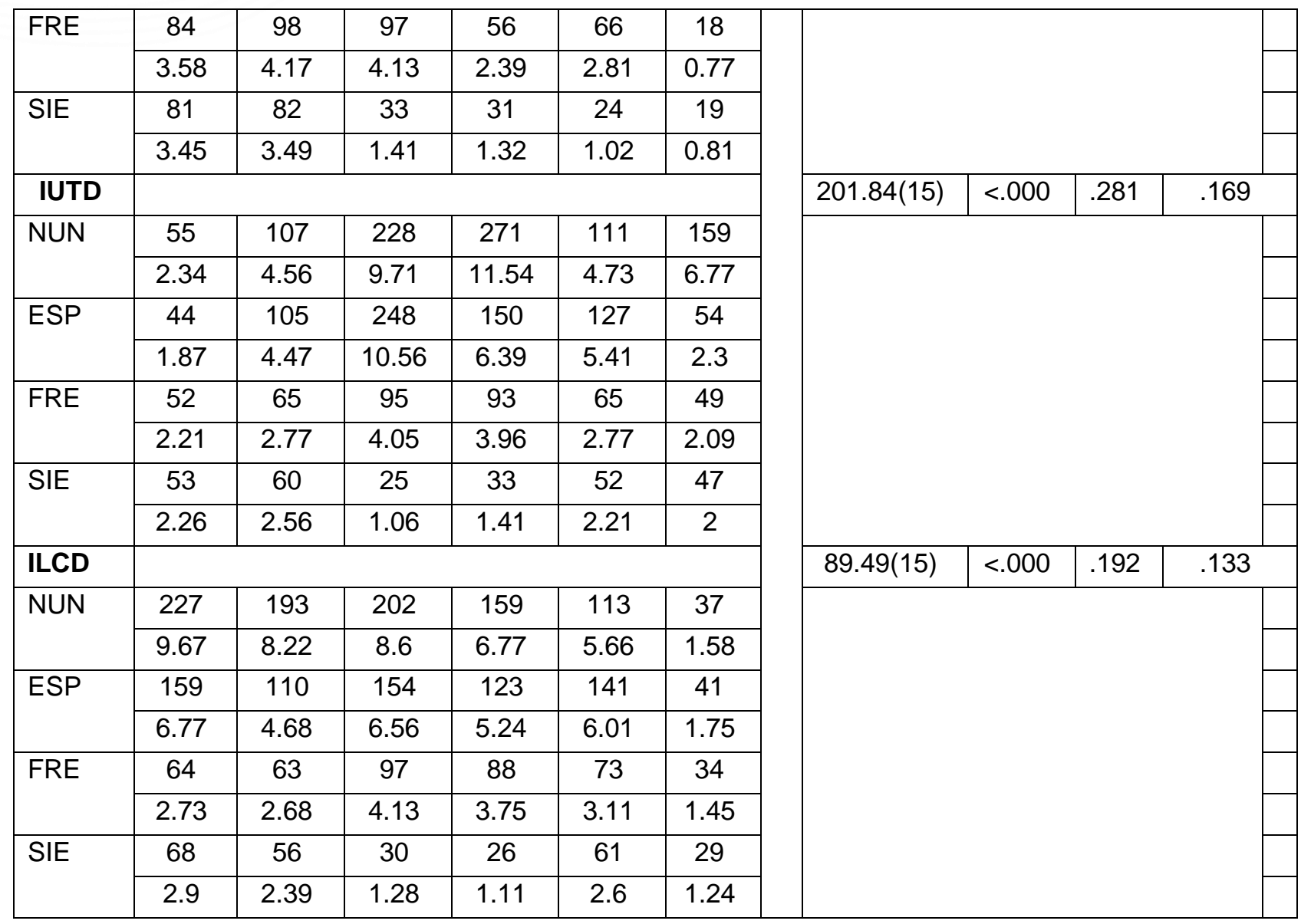

Nota: NUN=Nunca; ESP=Esporádico; FRE=Frecuente; SIE=Siempre.

La relación que mantienen las variables que conforman la competencia digital docente, determinadas en este caso como variables independientes, sobre la frecuencia de uso de la realidad aumentada, establecida como variable dependiente (Tabla 8), alcanzan valores de significancia, según los estadísticos empleados en el modelo de regresión múltiple $[F(260.579)=79.025 ; p<0.001]$, revelando el $70.7 \%$ de la varianza. Todas las variables muestran relación de significancia excepto netiqueta, desarrollo de contenidos digitales y datos personales de la salud. El resto de variables muestran relación de significancia con tendencias distintas. Las variables que reflejan un efecto significativo y de forma directa son almacenamiento y recuperación de datos y contenidos digitales, interacción mediante las tecnologías digitales, colaboración mediante canales digitales, programación, protección de datos personales e identidad digital, protección del entorno, resolución de problemas técnicos, identificación de necesidades y respuesta tecnológica e identificación de lagunas en la competencia digital. En cambio, aquellas que mantienen una relación inversamente proporcional son navegación, búsqueda, filtrado de información, datos y contenidos digitales, información, datos y contenidos digitales, compartir información y contenidos digitales, participación en línea, gestión de la identidad digital, derechos de autor y licencias, protección de dispositivos e innovación y uso de la tecnología digital de forma creativa.

Tabla 8

Efecto de las variables independientes sobre REAU

\begin{tabular}{|l|r|r|r|}
\hline Variables & $\boldsymbol{|}$ & $\boldsymbol{t}$ \\
\hline CD1 & \multicolumn{3}{|c|}{$\boldsymbol{p}$-valor } \\
\hline NBFI & $-.373(.049)$ & -7.598 & .000 \\
\hline IDCD & $-.493(.051)$ & -9.713 & .000 \\
\hline
\end{tabular}




\begin{tabular}{|c|c|c|c|}
\hline ARID & $.122(.039)$ & 3.151 & .002 \\
\hline \multicolumn{4}{|l|}{ CD2 } \\
\hline IMTD & $.514(.038)$ & 13.415 & .000 \\
\hline CICD & $-.614(.039)$ & -15.777 & .000 \\
\hline PCL & $-.205(.036)$ & -5.721 & .000 \\
\hline CMCD & $.311(.032)$ & 9.694 & .000 \\
\hline NETI & $-.050(.040)$ & -1.261 & .207 \\
\hline GIDI & $-.129(.041)$ & -3.116 & .002 \\
\hline \multicolumn{4}{|l|}{ CD3 } \\
\hline DCDI & $-.055(.044)$ & -1.258 & .209 \\
\hline IRCD & $.298(.041)$ & 7.290 & .000 \\
\hline DALI & $-.084(.037)$ & -2.260 & .024 \\
\hline PROG & $.177(.037)$ & 4.740 & .000 \\
\hline \multicolumn{4}{|l|}{ CD4 } \\
\hline PRDI & $-.727(.035)$ & -20.759 & .000 \\
\hline PDPE & $.157(.041)$ & 3.872 & .000 \\
\hline PRSA & $.018(.038)$ & .471 & .638 \\
\hline PREN & $.372(.044)$ & 8.396 & .000 \\
\hline \multicolumn{4}{|l|}{ CD5 } \\
\hline REPT & $.219(.037)$ & 5.938 & .000 \\
\hline INRT & $.234(.036)$ & 6.595 & .000 \\
\hline IUTD & $-.120(.034)$ & -3.578 & .000 \\
\hline ILCD & $.286(.032)$ & 8.846 & .000 \\
\hline
\end{tabular}

\section{Discusión y conclusiones}

Esta investigación ha tenido como objetivo conocer y analizar la incidencia del nivel de competencia digital docente en el empleo de la realidad aumentada como recurso didáctico para la impartición de materias relacionadas con las ciencias. Es incuestionable negar que las exigencias y características de la sociedad actual hacen ineludible la necesidad de hacer uso de la tecnología de forma creativa para innovar en los procesos de enseñanza aprendizaje (Asongu y Odhiambo, 2019; Avidov-Ungar y Hanin-Itzak, 2019; FuentesCabrera et al., 2019; Rodríguez-García, Raso y Ruiz, 2019; Starkey, 2019). De hecho, se constata como una demanda propia de la sociedad del conocimiento donde nos encontramos, así como en un escenario actual donde la pandemia de la COVID-19 ha exigido que los docentes se tengan que poner al día sobre este tipo de recursos y pasando de una enseñanza mayormente presencial a un escenario virtual o semipresencial.

Esta situación ha puesto de manifiesto algunas carencias de los sistemas educativos actuales, así como de los propios docentes y personal de administración y servicios debido, por un lado, a la escasez de recursos y, por otro lado, a la baja capacitación en el manejo de las tecnologías digitales.

Es por ello que, para el uso eficaz de las TIC aplicadas a la educación se requiere el desarrollo de una mejor competencia digital docente (Hilli, 2019 y Rodríguez-García, 2019), dado que los niveles de destrezas actuales continúan siendo todavía deficitarios por parte de esta población, tal y como mencionan multitud de investigaciones relacionadas, tales como Cuartero, Espinosa y Porlán (2019), Ewais y Troyer (2019), GisbertCervera y Lázaro-Cantabrana (2015), Lázaro et al. (2015), Rodríguez-García (2019) y Silva-Quiroz (2017). Además, tal y como hemos encontrado en este estudio, la competencia digital se sitúa en un nivel intermedio 
en los profesores de enseñanza secundaria de Andalucía, cifras que vienen confirmando lo que otros estudios, como los citados anteriormente, han mencionado hasta el momento.

A pesar de todo, debemos ser conscientes de que la innovación educativa -en no pocas ocasiones- va unida de la mano de la tecnología y, a su vez, de un buen desarrollo de habilidades y destrezas digitales que nos permitan relacionarnos eficazmente con la sociedad actual, tal como indicaron Canfarotta y Casado-Muñoz (2019) y Juhanak et al. (2019). Este es el caso, pues, del empleo de la RA como recurso didáctico que apoya el proceso de enseñanza-aprendizaje, la cual está adquiriendo cada vez más protagonismo en las aulas y en las diferentes etapas educativas (Barroso-Osuna et al., 2019; Bhagat et al., 2019; García-Bonete et al., 2019; Hendajani et al., 2019; Tzima et al., 2019; Villalustre et al., 2019). Este recurso, por un lado, sirve para acercar a los estudiantes a experiencias de aprendizaje más inmersivas y significativas (Hendajani et al., 2019). De igual modo, se consigue contextualizar más los aprendizajes y generar entornos activos de enseñanza donde el alumnado sea más protagonista y adquiera un papel más activo en lugar de ser receptor meramente pasivo de la enseñanza (Barroso-Osuna et al., 2019; García-Bonete et al., 2019).

Más concretamente, en relación a la investigación que aquí hemos presentado, hemos podido observar una relación de significancia entre el uso de la realidad aumentada y las distintas áreas de la competencia digital, por lo que se hace necesaria un nivel intermedio-alto en todas sus dimensiones para promover el uso de recursos didácticos creativos y experiencias de aprendizaje significativas y más exitosas (Pozo-Sánchez, López-Belmonte, Rodríguez-García y López-Núñez, 2020).

En este sentido, las áreas competenciales donde los docentes de ciencias muestran un mayor nivel, en relación al uso de la RA, es la colaboración mediante canales digitales y la innovación y uso de la tecnología digital de forma creativa. En cambio, presentan niveles más bajos en programación, resolución de problemas técnicos y desarrollo de contenidos digitales.

Por otro lado, el uso de la RA tiene una mayor incidencia y una relación directa con el almacenamiento y recuperación de datos y contenidos digitales, interacción mediante las tecnologías digitales, colaboración mediante canales digitales, programación, protección de datos personales e identidad digital, protección del entorno, resolución de problemas técnicos, identificación de necesidades y respuesta tecnológica e identificación de lagunas en la competencia digital. Sin embargo, aquellas que mantienen una relación inversamente proporcional son navegación, búsqueda, filtrado de información, datos y contenidos digitales, información, datos y contenidos digitales, compartir información y contenidos digitales, participación en línea, gestión de la identidad digital, derechos de autor y licencias, protección de dispositivos e innovación y uso de la tecnología digital de forma creativa. De todas ellas, donde hay una mayor fuerza de asociación es en la interacción mediante las tecnologías digitales, pudiendo ser considerada el área a desarrollar en los docentes que quieran hacer uso de la realidad aumentada.

Por tanto, podemos concluir afirmando que los docentes que utilizan la realidad aumentada en su praxis docente tienden a mostrar un nivel de competencia digital superior en contraposición con aquellos que dicen no usarla. Sin embargo, debido al carácter innovador de esta investigación, se precisa de una extrapolación a otros contextos donde se estudie la influencia de la competencia digital y la RA en los procesos educativos relacionados con la enseñanza de las ciencias, ampliando así el conocimiento científico al respecto.

\section{Referencias}

Agreda, M., Hinojo, M.A., y Sola, J.M. (2016). Design and validation of an instrument for assess digital skills of teachers in Spanish higher education. Píxel-Bit. Revista de Medios y Educación, (49), 39-56. https://doi.org/10.12795/pixelbit.2016.i49.03

Asongu, S. A., y Odhiambo, N. M. (2019). Enhancing ICT for quality education in sub-Saharan Africa. Education and Information Technologies, 24(5) 1-17. https://doi.org/10.1007/s10639-019-09880-9

Avidov-Ungar, O., y Hanin-Itzak, L. (2019). Sense of Empowerment Among School ICT Coordinators: Personal, SubjectArea and Leadership Empowerment. Technology, Knowledge and Learning, 24(3), 401417. https://doi.org/10.1007/s10758-017-9346-8

Barroso-Osuna, J., Gutiérrez-Castillo, J.J., Llorente-Cejudo, M.D., y Valencia-Ortiz, R. (2019). Difficulties in the Incorporation of Augmented Reality in University Education: Visions from the Experts. Journal of New Approaches in Educational Research, 8(2): 126-141. https://doi.org/10.7821/naer.2019.7.409

Bhagat, K. K., Liou, W. K., Michael Spector, J., y Chang, C. Y. (2019). To use augmented reality or not in formative assessment: a comparative study. Interactive Learning Environments, 27(5-6), 830-840. https://doi.org/10.1080/10494820.2018.1489857

Blayone, T. J. (2019). Theorising effective uses of digital technology with activity theory. Technology, Pedagogy and Education, 28(4) 1-16. https://doi.org/10.1080/1475939X.2019.1645728 
Bos, A., Herpich, F., Kuhn, I., Guarese, R.L., Tarouco, L. M., Zaro, MA, Pizzato, M., y Wives, L. (2019). La tecnología educativa y sus contribuciones en el enfoque y la atención de los estudiantes con respecto a los entornos de realidad aumentada $y$ el uso de sensores. Journal of Educational Computing Research, 57(7), 1832-1848. https://doi.org/10.1177/0735633119854033

Canfarotta, D., y Casado-Muñoz, R. (2019). Italian and Spanish Students' Perception on Use of Technology in Classrooms of Classics in Secondary School. Education in the Knowledge Society, 20, 1 14. https://doi.org/10.14201/eks2019_20_a13

Chen, Y. C. (2019). Effect of Mobile Augmented Reality on Learning Performance, Motivation, and Math Anxiety in a Math Course. Journal of Educational Computing Research, 57(7), 1695-1722. https://doi.org/10.1177/0735633119854036

Cuartero, M. D., Espinosa, M. P. P., y Porlán, I. G. (2019). Certificación de la Competencia Digital Docente: propuesta para el profesorado universitario. RIED. Revista Iberoamericana de Educación a Distancia, 22(1), 187-205. https://doi.org/10.5944/ried.22.1.22069

Erbas, C., y Demirer, V. (2019). The effects of augmented reality on students' academic achievement and motivation in a biology course. Journal of Computer Assisted Learning, 35(3), 450-458. https://doi.org/10.1111/jcal.12350

Ewais, A., y Troyer, O. D. (2019). A Usability and Acceptance Evaluation of the Use of Augmented Reality for Learning Atoms and Molecules Reaction by Primary School Female Students in Palestine. Journal of Educational Computing Research, 57(7), 1643-1670. https://doi.org/10.1177/0735633119855609

Falcó-Boudet, J. M. (2017). Evaluación de la competencia digital docente en la Comunidad Autónoma de Aragón. Revista electrónica de investigación educativa, 19(4), 73-83. https://doi.org/10.24320/redie.2017.19.4.1359

Fernández-Batanero, J.M., Cabero, J., y López, E. (2019). Knowledge and degree of training of primary education teachers in relation to ICT taught to students with disabilities. British Journal of Educational Technology, 50(4), 1961-1978. https://doi.org/10.1111/bjet.12675

Fuchsova, M., y Korenova, L. (2019). Visualisation in Basic Science and Engineering Education of Future Primary School Teachers in Human Biology Education Using Augmented Reality. European Journal of Contemporary Education, 8(1), 92102. https://doi.org/10.13187/ejced.2019.1.92

Fuentes-Cabrera, A., López-Belmonte, J., y Pozo-Sánchez, S. (2019). Análisis de la competencia digital docente: Factor clave en el desempeño de pedagogías activas con Realidad Aumentada. REICE: Revista lberoamericana sobre Calidad, Eficacia y Cambio en Educación, 17(2), 27-42. https://doi.org/10.15366/reice2019.17.2.002

Garcia-Bonete, M. J., Jensen, M., y Katona, G. (2019). A practical guide to developing virtual and augmented reality exercises for teaching structural biology. Biochemistry and Molecular Biology Education, 47(1), 16-24. https://doi.org/10.1002/bmb.21188

Gisbert-Cervera, M., y Lázaro-Cantabrana, J.L. (2015). Desarrollo profesional en competencia digital docente y mejora de la calidad escolar desde la perspectiva docente: un estudio de caso. Journal of New Approaches in Educational Research 4(2), 115-122. https://doi.org/10.7821/naer.2015.7.123

Hendajani, F., Hakim, A., Sudiro, S. A., Saputra, G. E., y Ramadhana, A. P. (2019). Tracking Visualization Of 3 Dimensional Object Natural Science Learning Media In Elementary School With Markerless Augmented Reality Based On Android. Journal of Physics: Conference Series, 1192(1), 1-8. https://doi.org/10.1088/1742-6596/1192/1/012055 Hernández, R., Fernández, C., y Baptista, M.P. (2014). Metodología de la investigación. México D.F.: McGrawHill Education.

Hilli, C. (2019). Extending classrooms through teacher collaboration in Virtual Learning Environments. Educational Action Research, 1-16. https://doi.org/10.1080/09650792.2019.1654901

Hsu, H. P., Wenting, Z., y Hughes, J. E. (2019). Developing elementary students' digital literacy through augmented reality creation: Insights from a longitudinal analysis of questionnaires, interviews, and projects. Journal of Educational Computing Research, 57(6), 1400-1435. https://doi.org/10.1177/0735633118794515

Instefjord, E.J., y Munthe, E. (2017). Educating digitally competent teachers: A study of integration of professional digital competence in teacher education. Teaching and teacher education, 67, 37-45. https://doi.org/10.1016/j.tate.2017.05.016 INTEF. (2017). Marco de Competencia Digital. Madrid: Ministerio de Educación, Ciencia y Deportes. Recuperado de https://aprende.intef.es/sites/default/files/2018-05/2017_1020_Marco-Com\%C3\%BAn-de-Competencia-Digital-

Docente.pdf

Juhanak, L. Zounek, J. Zaleska, K. Barta, O., y VIckova, K. (2019). The relationship between the age at first computer use and students' perceived competence and autonomy in ICT usage: A mediation analysis. Computers y Education, 141, 114. https://doi.org/10.1016/j.compedu.2019.103614

Kularbphettong, K., y Puengpo, N. (2018). The Effective of Enhancing Classroom Learning Through Augmented Reality. Advanced Science Letters, 24(11), 7917-7919. https://doi.org/10.1166/asl.2018.12456

Lázaro, J., Usart, M., y Gisbert, M. (2019). Assessing Teacher Digital Competence: the Construction of an Instrument for Measuring the Knowledge of Pre-Service Teachers. Journal of New Approaches in Educational Research (NAER Journal), 8 (1), 73-78. Recuperado de: https://www.learntechlib.org/p/207150/.

López-Gómez, E. (2018). The Delphi method in current educational research: a theoretical and methodological review. Educación XX1, 21(1), 17-40. https://doi.org/10.5944/educXX1.15536

Mustami, M. K., Syamsudduha, S., Safei, y Ismail, M. I. (2019). Validity, practicality, and effectiveness development of biology textbooks integrated with augmented reality on high school students. International Journal of Technology Enhanced Learning, 11(2), 187-200. https://doi.org/10.1504/IJTEL.2019.098789 
Olofsson, A. D., Fransson, G., y Lindberg, J. O. (2019). A study of the use of digital technology and its conditions with a view to understanding what 'adequate digital competence'may mean in a national policy initiative. Educational Studies, 117. https://doi.org/10.1080/03055698.2019.1651694

Pozo-Sánchez, S., López-Belmonte, J., Rodríguez-García, A. M., \& López-Núñez, J. A. (2020). Teachers' digital competence in using and analytically managing information in flipped learning (Competencia digital docente para el uso y gestión analítica informacional del aprendizaje invertido). Culture and Education, 32(2), 213-241.

Prendes-Espinosa, M. P., Gutierrez-Porlan, I., y Martinez-Sanchez, F. (2018). Digital competence: a need for university teachers in the 21st century. RED-Revista de Educación a distancia, 56, 7578-7680. https://doi.org/10.6018/red/56/7 Rodríguez-García, A. M. (2019). Análisis de competencias digitales adquiridas en el grado de educación primaria y su adecuación para el desempeño de una labor docente de calidad en Andalucía (Tesis Doctoral). Universidad de Granada.

Rodríguez García, A. M., Raso Sánchez, F., \& Ruiz Palmero, J. (2019). Competencia digital, educación superior y formación del profesorado: un estudio de meta-análisis en la Web of Science. Pixel-Bit: Revista de Medios y Educación, 54, 65-81.

Safadel, P., y White, D. (2019). Facilitating molecular biology teaching by using augmented reality (AR) and protein data bank (PDB). TechTrends, 63(2), 188-193. https://doi.org/10.1007/s11528-018-0343-0

Salcines, I., y González, N. (2016). Design and Validation of the Smartphone and University. Lectures' view (SUOL) questionnaire. Revista Complutense de Educación, 27(2), 603-632. https://doi.org/10.5209/rev_RCED.2016.v27.n2.46912

Santos, M.A., Sotelino, A., Jover, G., Álvarez-Castillo, J.L., y Vázquez, V. (2017). Design and validation of a questionnaire on Universiry teaching practice and attitudes towards innovation (CUPAIN). Educación XX1, $20(2), 39-71$. https://doi.org/10.5944/educXX1.17806

Silva-Quiroz, J. (2017). Insertion of ICT in pedagogies in the humanities area at a Chilean university. Psicología, Conocimiento y Sociedad, 7(2), 110-133. https://doi.org/10.26864/PCS.v7.n2.6

Starkey, L. (2019). Una revisión de la investigación que explora la preparación docente para la era digital. Cambridge Journal of Education, 1-20. https://doi.org/10.1080/0305764X.2019.1625867

Tomara, M., y Gouscos, D. (2019). Un estudio de caso: visualizar las fuerzas de Coulomb con la ayuda de la realidad aumentada. Journal of Educational Computing Research, 57(7) 1626-1642. https://doi.org/10.1177/0735633119854023 Tzima, S., Styliaras, G., y Bassounas, A. (2019). Augmented Reality Applications in Education: Teachers Point of View. Education Sciences, 9(2), 99. https://doi.org/10.3390/educsci9020099

Villalustre, L., Del Moral, M. E., y Neira, M. R. (2019). Percepción docente sobre la Realidad Aumentada en la Enseñanza de Ciencias en Primaria. Análisis DAFO. Revista Eureka sobre Enseñanza y Divulgación de las Ciencias, 16(3), 33013301. https://doi.org/10.25267/Rev_Eureka_ensen_divulg_cienc.2019.v16.i3.3301 